\title{
ORIGINAL ARTICLE \\ Association of dietary patterns and hyperuricemia: a cross-sectional study of the Yi ethnic group in China
}

\author{
Xirun Liu', Shanshan Huang', Wangdong Xu', Aijing Zhou², Hui Li', Rong Zhang', Ya Liu', \\ Yan Yang' and Hong Jia'**
}

ISchool of Public Health, Southwest Medical University, Luzhou, China; ${ }^{2}$ Department of Medical Record, Sichuan

University West China Hospital, Chengdu, China

\section{Abstract}

Background: Diet plays an important role in the development of hyperuricemia (HUA), but evidence for association between overall dietary patterns and HUA is scarce and inconsistent. The present study aims to explore association of dietary patterns and HUA among the Yi ethnic group of China.

Methods: This is a cross-sectional study involving people aged more than 18 years. Principal component factor analysis (PCFA) on food groups from a semi-quantitative 52-item food frequency questionnaire was applied to identify dietary patterns. HUA status was regressed on tertiles of factor scores to estimate prevalence ratio (PR) by using log-binomial model.

Results: Of the 1,893 participants (18-96 years), 398 (21.0\%) were diagnosed with HUA. Three dietary patterns were identified: 'plant-based', 'animal products', and 'mixed food'. The 'animal products' was characterized by high intake of fish, animal giblets, fresh meat, and wheat products. After adjustment for potential confounders, the highest tertile of 'animal products' pattern score was associated with higher prevalence of HUA when compared with the lowest tertile (PR: 1.34, 95\% CI: 1.06-1.70). The other two patterns were not related to HUA.

Conclusions: 'Animal products' dietary pattern was correlated with HUA among the Yi ethnic group of China.

Keywords: dietary pattern; hyperuricemia; factor analysis; cross-sectional study; the Yi ethnic group

To access the supplementary material, please visit the article landing page

Received: 2 I September 20 17; Revised: 28 March 20 I8; Accepted: 29 March 20 18; Published: 25 April 2018

$\mathrm{H}$ yperuricemia (HUA) is a metabolic disease caused by purine metabolic abnormalities. It is characterized by increased formation or reduced excretion of serum uric acid (SUA). Most patients with HUA are asymptomatic and do not receive treatment. However, previous studies indicated that HUA is an important risk factor for hypertension (1), diabetes (2), cardiovascular disease (CVD) (3), metabolic syndrome (MetS) (4), and that it also features in the pathophysiology of gout (5) and renal damage $(6,7)$. In recent decades, the prevalence of HUA has significantly increased, accompanied with younger age $(8,9)$. The prevalence of HUA was increasing rapidly in China as well $(5,10)$.

Genetics, lifestyle, and diet are susceptible factors contributing to HUA. It is accepted that diet is the most modifiable factor $(11,12)$. In nutritional epidemiology, researches are mainly paying attention to single nutrient or food (13) and do not consider the complexity of diet, and interactions of nutrients or foods. Representative of comprehensive dietary variables, dietary pattern attempts to reveal the influence of overall diet. Hence, it predicts association of nutrition and diseases more effectively, and adjusting a comprehensive dietary pattern is more effective than adjusting intake of single food or nutrient in the disease prevention and control. Dietary patterns analysis has been applied to evaluate association of diet with Mets (14, 15), CVD (16), diabetes (17), and obesity (18). However, there are few studies about dietary patterns and HUA. In addition, available evidence did not show consistent results that which pattern is protective or risky for $\operatorname{HUA}(19,20)$.

Liangshan Yi Autonomous Prefecture is located in the southwestern Sichuan province, where the aboriginal Yi ethnic group lives a relatively primitive life and keeps its 
unique dietary culture and custom. The prevalence of HUA among adult Yi people was 14.71\% in 2007 (21), while the prevalence in rural area was up to $22.0 \%$ in 2014 (22), significantly higher than other areas $(5,23)$. Diet is related to the incidence of HUA, but whether the diet of Yi people renders the health of its people a challenge remains to be clarified. Therefore, this study aims to characterize their dietary patterns and assess the correlation of these patterns with the prevalence of HUA among the Yi ethnic group of China.

\section{Methods}

\section{Participants}

This is a cross-sectional study based on Yi people aged more than 18 years. A representative sample of the Yi ethnic group was obtained by a multistage stratified cluster sampling method. Details of survey methods and sample selection are described in our previous study (24). Of the 1,918 participants aged 18-96 years, 1,893 (98.70\%) were included for the analyses. Twenty-five of the participants were excluded because of no SUA measurement or more than $50 \%$ of the dietary questions being incomplete. Exclusion criteria were (1) renal failure, (2) medication for HUA or gout, (3) pregnancy, and (4) inability to communicate or walk normally. The corresponding protocol was approved by the Ethics of Research Committee of the Southwest Medical University. Informed consent was obtained from all participants.

\section{Data collection}

Data collection was accomplished by local medical workers, well-trained investigators, and field workers. Details of the procedure are as follows:

\section{Questionnaire survey}

Data collection was performed by face-to-face interviews with interviewer-administered questionnaires. Information on socio-demographic characteristics and other potential confounders such as age, gender, years of education ( $\leq 6$ years, $7-12$ years, $\geq 13$ years), drinking and smoking status (yes or no) and presence of hypertension was collected using a structured questionnaire. A semi-quantitative 52-item food frequency questionnaire (FFQ) was used to measure habitual diet over the past year. The FFQ included questions on the types of food items, frequency (daily, weekly, monthly, yearly, or never), and amount (portion size in grams per time) of food consumption. Total alcohol consumption was the sum of daily intake of different alcoholic beverages. Consumption of cooking oil of a family was recorded and averaged for assessing the individual's cooking oil consumption per day. The FFQ was designed based on culture-specific dishes, recipes, and tested on a local sample to check for clarity and applicability under the Yi culture.

\section{Physical examination}

Heights were measured to the nearest $0.1 \mathrm{~cm}$ using a secure standard height measuring equipment while wearing no shoes. Weight was collected using a calibrated electronic digital scale, accurate to $0.1 \mathrm{~kg}$ with subjects wearing only light underwear. Body mass index (BMI) was calculated by weight $(\mathrm{kg}) /$ height $^{2}\left(\mathrm{~m}^{2}\right)$. Waist circumference (WC) was measured with a tape measure to the nearest $0.1 \mathrm{~cm}$ around the midpoint between the lowest rib and the iliac. Hip circumference was measured to the nearest $0.1 \mathrm{~cm}$ with the soft tape placed around the symphysis pubis and the posterior gluteus maximus. Waist-hip ratio (WHR) was calculated by WC $(\mathrm{cm}) /$ hip circumference $(\mathrm{cm})$. Blood pressure (BP) was measured by electronic sphygmomanometer after participant rested for $5 \mathrm{~min}$.

\section{Laboratory test}

Fasting blood samples were collected from each individual and sent to Liangshan center for disease control and prevention. SUA, fasting plasma glucose (GLU), triglycerides (TG), total cholesterol (TC), high-density lipoprotein cholesterol (HDL-C), and lowdensity lipoprotein cholesterol (LDL-C) were measured by an automatic biochemical analyzer (Mindary, BS-820, Shenzhen, China).

\section{Definition of disease}

HUA was diagnosed when SUA $\geq 420 \mu \mathrm{mol} / \mathrm{L}(7.0 \mathrm{mg} / \mathrm{dL})$ for men, and SUA $\geq 360 \mu \mathrm{mol} / \mathrm{L}(6.0 \mathrm{mg} / \mathrm{dL})$ for women (25). Hypertension: BP $\geq 140 / 90 \mathrm{mmHg}$, or usage of antihypertensive drugs. Hyperlipemia: if one or more of the following criteria were satisfied: (1) $\mathrm{TC}>5.72 \mathrm{mmol} / \mathrm{L}$, (2) $\mathrm{TG}>1.70 \mathrm{mmol} / \mathrm{L}$, and (3) $\mathrm{HDL}-\mathrm{C}<1.3 \mathrm{mmol} / \mathrm{L}$ in women and $<1.0 \mathrm{mmol} / \mathrm{L}$ in men (26).

\section{Assessment of dietary intake}

Food items were categorized into 18 food groups based on Food Composition Table, similarities in ingredients, and purine contents (Supplementary Table 1). The total intake of food group depended on summing the daily gram intake of each food item within the group. Principal component factor analysis (PCFA) with varimax orthogonal rotation was applied. The number of patterns was determined by the cumulative contribution rate of variance, eigenvalue $>1$, and the interpretability of the results. Food groups with rotated factor loading greater than 0.35 were regarded as main components of each pattern. Dietary pattern scores were calculated by summing the product of standardized intake of each group multiplied by regression coefficients and were categorized into tertiles. 


\section{Statistical analysis}

Frequencies (\%), means \pm standard deviations (SD) were used to describe various socio-demographic characteristics, lifestyle, anthropometric measurement, and clinical characteristics of participants in the nutrition survey. Binary HUA status was regressed on tertiles of factor scores to obtain prevalence ratios (PRs) by using logbinomial model. All the analyses were conducted using the SAS 9.3 software and two-tailed and considered statistically significant when $p<0.05$.

\section{Results}

\section{Characteristics of participants}

A total of 1,893 participants were included, and the prevalence of HUA was $21.0 \%$. Characteristics of this population were listed in Table 1. Participants with and without
HUA showed significant differences in terms of gender, education, smoking, and drinking status. Compared with those without HUA, participants with HUA showed higher expression of TG, TC, and SUA, and higher BMI, WC, WHR, and BP.

\section{Extraction of dietary patterns}

We identified three dietary patterns in this population, which we named: a 'plant-based' dietary pattern, as it was characterized by high intake of vegetables, fruits, mushroom, algae food, legumes, nuts, brawn, and bacon; an 'animal products' dietary pattern, which was characterized by high intake of fish, animal giblets, fresh meat, wheat products; and a 'mixed food' dietary pattern, as it was mostly composed of rice, cereal, tubers, snack, dessert, eggs, and animal giblets (Table 2). The three patterns explained $34.22 \%$ of total variance, with the 'plant-based',

Table 1. Demographics, lifestyle characteristics, anthropometric measurements, and serum biochemical_indexes of Yi People participants in the nutrition survey $(n=1,893)$

\begin{tabular}{|c|c|c|c|}
\hline \multirow[t]{2}{*}{ Variables } & \multirow{2}{*}{$\begin{array}{c}\text { Participants with HUA } \\
n=398\end{array}$} & \multirow{2}{*}{$\begin{array}{c}\text { Participants without HUA } \\
n n=1,495\end{array}$} & \multirow[t]{2}{*}{$p$} \\
\hline & & & \\
\hline Gender & & & $<0.001$ \\
\hline Male & $270(67.8)$ & $636(42.5)$ & \\
\hline Female & $128(32.2)$ & $859(57.5)$ & \\
\hline Age group & & & 0.247 \\
\hline $18-44$ & $244(6 \mid .3)$ & $847(56.7)$ & \\
\hline $45-59$ & $98(24.6)$ & $415(27.7)$ & \\
\hline$\geq 60$ & $56(14.1)$ & $233(15.6)$ & \\
\hline Years of education & & & $<0.001$ \\
\hline$\leq 6$ & $227(57.0)$ & I,I 62 (77.7) & \\
\hline $7-12$ & 138 (34.7) & $274(18.3)$ & \\
\hline$\geq 13$ & $33(8.3)$ & $59(3.9)$ & \\
\hline Smoking & & & $<0.001$ \\
\hline Yes & $170(42.7)$ & $486(32.5)$ & \\
\hline No & $228(57.3)$ & $\mathrm{I}, 009(67.5)$ & \\
\hline Drinking & & & $<0.001$ \\
\hline Yes & $231(58.0)$ & $723(48.4)$ & \\
\hline No & $167(42.0)$ & $772(5 \mathrm{I} .6)$ & \\
\hline BMI $\left(k g / m^{2}\right)$ & $23.58 \pm 3.57$ & $22.25 \pm 3.16$ & $<0.001$ \\
\hline Waistline & $83.46 \pm 10.61$ & $78.66 \pm 9.43$ & $<0.001$ \\
\hline WHR & $0.88 \pm 0.07$ & $0.86 \pm 0.07$ & $<0.001$ \\
\hline $\mathrm{SBP}(\mathrm{mmHg})$ & $131.95 \pm 18.96$ & $125.59 \pm 17.68$ & $<0.001$ \\
\hline $\mathrm{DBP}(\mathrm{mmHg})$ & $79.86 \pm 13.78$ & $76.09 \pm 12.23$ & $<0.001$ \\
\hline GLU (mmol/L) & $5.57 \pm 1.46$ & $5.59 \pm 1.66$ & 0.819 \\
\hline $\mathrm{TC}(\mathrm{mmol} / \mathrm{L})$ & $5.13 \pm 1.08$ & $4.89 \pm 0.97$ & $<0.001$ \\
\hline TG (mmol/L) & $1.79 \pm 1.27$ & $1.37 \pm 1.13$ & $<0.001$ \\
\hline
\end{tabular}

Arithmetic mean values and standard deviations (SD) for continuous variables; number of participants and percentages for categorical variables; test for differences in the characteristics of the participants with and without HUA were taken using the Chi-square test for categorical variables and the ANOVA analyses for continuous variables.

BMI: body mass index $=$ weight $(\mathrm{kg}) /$ height ${ }^{2}\left(\mathrm{~m}^{2}\right)$;WHR: waist-hip ratio $=$ waistline $(\mathrm{cm}) / \mathrm{hipline}(\mathrm{cm}) ;$ SBP: systolic blood pressure; DBP: diastolic blood pressure; SUA: serum uric acid; HUA: hyperuricemia; GLU: glucose; TC: serum cholesterol;TG: serum triglycerides. 
Table 2. Dietary pattern factor loading matrix of the Yi people

\begin{tabular}{lccc}
\hline Food groups & Plant-based & Animal products & Mixed food \\
\hline Mushroom and algae food & $0.672^{\mathrm{a}}$ & - & - \\
Vegetables & 0.613 & - & - \\
Legumes & 0.518 & - & 0.365 \\
Nuts & 0.506 & - & - \\
Brawn, bacon & 0.486 & - & - \\
Fruits & 0.372 & - & - \\
Sugary beverages & - & - & - \\
Dairy & - & - & - \\
Oil & - & - & - \\
Alcoholic beverages & - & - \\
Wheat and its products & - & 0.856 & - \\
Fish & - & 0.847 & - \\
Fresh meat & - & 0.472 & - \\
Snacks and dessert & 0.357 & - & 0.624 \\
Animal giblets & - & 0.458 & 0.612 \\
Other cereal and tubers & - & - & 0.574 \\
Eggs & - & - & 0.458 \\
Rice & 12.27 & - & 0.409 \\
Contribution rate (\%) & -2.27 & 23.75 & 10.47 \\
The cumulative contribution rate (\%) & & 34.22 \\
\hline
\end{tabular}

${ }^{\mathrm{a} F a c t o r}$ loading $>0.35$ are listed.

${ }^{\mathrm{b}} \mathrm{Gram}$ or Liang.

'animal products', and mixed food' constituting 12.27\%, $11.48 \%$, and $10.47 \%$, respectively, of the total variance. Food intake across tertiles of each dietary pattern was listed (Supplementary Table 2).

\section{Association between dietary patterns and HUA}

Higher adherence to 'animal products' pattern was related to higher prevalence of HUA (crude model: $p$-trend $=$ 0.027). After adjustment for confounders, the association between this pattern and HUA was still significant. (the highest tertile: $\mathrm{PR}=1.34 ; 95 \% \mathrm{CI}: 1.06-1.70 ; p$-trend $=$ $0.031)$. The other two patterns, 'plant-based' and 'mixed food', were not associated with HUA (Table 3).

\section{Discussion}

In this study, three major dietary patterns, 'plant-based', 'animal products', and 'mixed food' were identified. The 'animal products' pattern was positively correlated with HUA. However, the other two patterns showed no association with HUA.

The 'animal products' pattern was characterized by high intake of fish, fresh meat, animal giblets, and legumes. To date, several studies have discussed the association of diet and HUA. The 'animal product and fried food' pattern, which indicated high consumption of pork, eggs, animal, poultry, and fried wheat, was associated with HUA (27). Tsai et al. reported that uric acid-prone pattern, including seafood, meat, beverages, organ meat, eggs, was positively related to concentration of SUA (19). Zykova et al. reported that high intake of meat was associated with elevated SUA, and intake of fish was not associated with high levels of SUA (13). Some other studies indicated that high intake of red meat, animal organ, and seafood are related to elevated levels of SUA and an increased risk of gout, because these food were purine-rich foods, and excessive purine intake may increase the levels of SUA $(28,29)$ and cause HUA $(30,31)$. In addition, high consumption of animal products, especially red meat, was often accompanied with excessive intake of calorie and fat, which may lead to obesity or centripetal obesity. HUA was associated with obesity $(29,32)$, and the incidence of HUA was increased accompanied with elevated BMI (29). Besides, centripetal obesity was a powerful stimulus for increasing the plasma insulin level and, therefore, it may increase the risk of HUA (33). In this study, we found that BMI of participants in the HUA group was significantly higher than that of the non-HUA group, but there was no significant difference in BMI among people from different dietary patterns, possibly suggesting that the high prevalence of HUA in the Yi people was mainly attributed to their diet. Collectively, our findings indicated that the prevalence of HUA was related to the 'animal products' pattern.

The 'plant-based' pattern was characterized by high intake of vegetables, fruits, legumes, nuts, brawn, 
Table 3. Analysis of the dietary patterns and HUA of the Yi people

\begin{tabular}{|c|c|c|c|c|c|c|}
\hline \multirow[t]{2}{*}{ Dietary patterns } & \multirow{2}{*}{$\frac{\text { Participants with HUA }}{n(\%)}$} & \multirow{2}{*}{$\frac{\text { SUA }(\mathrm{mmol} / \mathrm{L})}{\text { Median }}$} & \multicolumn{2}{|c|}{ Crude model } & \multicolumn{2}{|c|}{ Adjusted model } \\
\hline & & & PR $(95 \% \mathrm{Cl})$ & $p$ for trend & PR $(95 \% \mathrm{Cl})$ & $p$ for trend \\
\hline Plant-based & & & & 0.750 & & 0.763 \\
\hline TI & 124 (19.7) & 316.0 & Reference & & Reference & \\
\hline $\mathrm{T} 2$ & $|2|(19.2)$ & 323.0 & $0.94(0.75-1.18)$ & & $0.93(0.75-1.15)$ & \\
\hline T3 & $153(24.2)$ & 340.0 & $1.15(0.93-1.43)$ & & $1.03(0.84-1.26)$ & \\
\hline Animal products & & & & 0.027 & & 0.031 \\
\hline TI & $97(15.4)$ & 310.0 & Reference & & Reference & \\
\hline $\mathrm{T} 2$ & $130(20.6)$ & 313.0 & $1.32(1.03-1.69)^{*}$ & & $1.32(1.04-1.68)^{*}$ & \\
\hline $\mathrm{T} 3$ & |7| (27.I) & 354.0 & $1.69(1.34-2.14)^{*}$ & & $1.34(1.06-1.70)^{*}$ & \\
\hline Mixed food & & & & 0.157 & & 0.221 \\
\hline TI & $156(24.7)$ & 334.0 & Reference & & Reference & \\
\hline $\mathrm{T} 2$ & $124(19.7)$ & 326.0 & $0.85(0.69-1.05)$ & & $0.86(0.7|-| .05)$ & \\
\hline T3 & 118 (18.7) & 318.0 & $0.90(0.7|-I| 2)$. & & $0.97(0.78-1.20)$ & \\
\hline
\end{tabular}

Crude Model: No adjustment for any confounders.

Adjusted Model: Adjustment for age group (I8-44, 45-59, 60 years); gender (male/female); BMI: body mass index; smoking (smoker/non-smoker); drinking (drinking/no drinking); hypertension (with/without); hyperlipidemia (with/without).

The median value of each tertile was assigned to each subject in the same tertile and treated as a continuous variable in regression analysis. $* p<0.05$.

and bacon. Fruits and vegetables are rich in dietary fiber, vitamin $\mathrm{C}$, and folate, which were considered as protective factors for HUA $(13,34)$. On the contrary, some other studies did not suggest fruits and vegetables as protective factors $(30,35)$. Zhang et al. showed that fruit pattern, including soybean products, fruits, vegetables, and starchy tubers, was negatively related to HUA (27). A study among the Taiwanese indicated that vegetable and fruit pattern was not related to SUA concentration (20). High intake of vitamin C may reduce the SUA levels, owing to uricosuric effects mediated by inhibition of uric acid transporters 1 (URAT1) and/or sodiumdependent anion cotransport $(36,37)$. Fiber is possible to bind to SUA. One-third of the SUA produced every day is eliminated in intestinal secretions and saliva (27, 38). Our findings showed that the 'plant-based' pattern was not associated with HUA, where fruits and vegetables may decrease the risk of HUA. It is noteworthy that brawn and bacon heavily consumed in this pattern contributes to elevated SUA, increasing the risk of HUA (28, $30)$. Therefore, it is possible that the influence of purine and fat-rich foods may counteract the protective effects that fruits and vegetables provided for HUA, which may reflect the superiority of application of dietary patterns analysis.

'Mixed food' pattern, comprising rice, cereal, tubers, snack, dessert, eggs and animal giblets, was not associated with HUA. Cereal is an important source of vitamin $\mathrm{B}$ and is rich in carbohydrates, which are inversely correlated with levels of SUA $(13,34)$. High consumption of animal giblets was regarded as an adverse factor, resulting in elevated levels of SUA $(31,34)$. High consumption of snack and dessert contributed to overweight and upregulation of SUA levels (33). Studies about the association of SUA/HUA and animal protein were inconsistent. Zykova et al. reported that the high consumption of egg was associated with elevated levels of SUA (13). By contrast, Choi et al. showed that a higher intake of animal protein was not associated with an increased risk of gout (31), and consumption of animal protein was associated with higher prevalence of HUA (13). The overall effect of 'mixed food' pattern could be explained by the interaction of foods or nutrients. Further studies are needed to give more information about the interaction effects and confirm the result.

The present study has several strengths. First, questionnaires were administered by well-trained investigators and were not self-completed. This may lead to higher response rate, and provide convenience that illiterate people could also be included in the investigation. Second, collected dietary data over the past year could capture the picture of long-term dietary behaviors for individuals. Third, this study identified healthy or unhealthy dietary patterns and could form the starting point for development of new dietary guideline(s).

This study has several limitations. First, this is a crosssectional study, which may limit the ability to make a causal inference. Second, the sample size may not be sufficient enough; therefore, larger sample would be considered in further research. 


\section{Conclusion}

The study showed that 'animal products' pattern, characterized by high intake of fish, fresh meat, animal giblets, and wheat products, was correlated with increased prevalence of HUA in the Yi ethnic group of China. Our findings may aid in developing dietary guidelines for adults and providing the population with guidance for healthy dietary patterns based on the access to local culture and customs, and encourage people in the risky pattern to change their dietary structure to prevent and control HUA.

\section{Acknowledgements}

This on-site investigation has come to a successful conclusion. We would like to show our appreciation to the subjects who participated in this study for their cooperation and support, to Liangshan Prefecture CDC, teachers and students from Southwest Medical Universitythe, and the local health workers and village cadres for their effort and support. This study was supported by Danone. The funder had no role in the study design, analysis or interpretation of data, or in the writing of the manuscript. This study was registered in Chinese clinical trial registry (Registr ID: ChiCTR-ECS-14004115).

\section{Disclosure statement}

The authors declare no conflict of interest.

\section{Funding}

This work was supported by the Danone dietary nutrition research and education fund of Danone Institute (China) under (grant number DIC2013-03).

\section{References}

1. Yokokawa H, Fukuda H, Suzuki A, Fujibayashi K, Naito T, Uehara Y, et al. Association between serum uric acid levels/ hyperuricemia and hypertension among 85,286 Japanese workers. J Clin Hypertens 2016; 18(1): 53-9.

2. Krishnan E, Pandya BJ, Chung L, Hariri A, Dabbous O. Hyperuricemia in young adults and risk of insulin resistance, prediabetes, and diabetes: a 15-year follow-up study. Am J Epidemiol 2012; 176(2): 108-16.

3. Braga F, Pasqualetti S, Ferraro S, Panteghini M. Hyperuricemia as risk factor for coronary heart disease incidence and mortality in the general population: a systematic review and meta-analysis. Clin Chem Lab Med 2016; 54(1): 7-15.

4. Wei CY, Sun CC, Wei CC, Tai HC, Sun CA, Chung CF, et al. Association between hyperuricemia and metabolic syndrome: an epidemiological study of a Labor Force Population in Taiwan. Biomed Res Int 2015; 2015(7): 369179.

5. Rui L, Cheng H, Di W, Xia X, Gu J, Guan H, et al. Prevalence of hyperuricemia and gout in Mainland China from 2000 to 2014: a systematic review and meta-analysis. Biomed Res Int 2015; 2015(15 Suppl): 1-12.

6. Kuwata H, Okamura S, Hayashino Y, Ishii H, Tsujii S. Serum uric acid levels are associated with a high risk of rapid chronic kidney disease progression among patients with type 2 diabetes: a prospective cohort study [Diabetes Distress and Care Registry at Tenri (DDCRT 12)]. Diabetol Int 2016; 7(4): 1-9.

7. Edwards NL. The role of hyperuricemia and gout in kidney and cardiovascular disease. Cleve Clin J Med 2008; 75(Suppl 5): S13.

8. Zhu Y, Pandya BJ, Choi HK. Prevalence of gout and hyperuricemia in the US general population: the National Health and Nutrition Examination Survey 2007-2008. Arthritis Rheum 2011; 63(10): 3136.

9. Uaratanawong S, Suraamornkul S, Angkeaw S, Uaratanawong R. Prevalence of hyperuricemia in Bangkok population. Clin Rheumatol 2011; 30(7): 887.

10. Liu H, Zhang XM, Wang YL, Liu BC. Prevalence of hyperuricemia among Chinese adults: a national cross-sectional survey using multistage, stratified sampling. J Nephrol 2014; 27(6): 653-8.

11. Qiu L, Cheng XQ, Wu J, Liu JT, Xu T, Ding HT, et al. Prevalence of hyperuricemia and its related risk factors in healthy adults from Northern and Northeastern Chinese provinces. BMC Public Health 2013; 13(1): 664.

12. Sari I, Akar S, Pakoz B, Sisman AR, Gurler O, Birlik M, et al. Hyperuricemia and its related factors in an urban population, Izmir, Turkey. Rheumatol Int 2009; 29(8): 869-74.

13. Zykova SN, Storhaug HM, Toft I, Chadban SJ, Jenssen TG7, White SL. Cross-sectional analysis of nutrition and serum uric acid in two Caucasian cohorts: the AusDiab Study and the Tromsø study. Nutr J 2015; 14(1): 49. doi: 10.1186/ s12937-015-0032-1

14. Naja F, Nasreddine L, Itani L, Adra N, Sibai AM, Hwalla N. Association between dietary patterns and the risk of metabolic syndrome among Lebanese adults. Eur J Nutr 2013; 52(1): 97-105.

15. Mazidi M, Pennathur S, Afshinnia F. Link of dietary patterns with metabolic syndrome: analysis of the National Health and Nutrition Examination Survey. Nutr Diabetes 2017; 7(3): e255.

16. Rodríguez-Monforte M, Flores-Mateo G, Sánchez E. Dietary patterns and CVD: a systematic review and meta-analysis of observational studies. Br J Nutr 2015; 114(9): 1341-59.

17. Batis C, Mendez MA, Gordonlarsen P, Sotres-Alvarez D, Adair L, Popkin B. Using both principal component analysis and reduced rank regression to study dietary patterns and diabetes in Chinese adults. Public Health Nutr 2016; 19(2): 195.

18. Paradis AM, Godin G, Pérusse L, Vohl MC. Associations between dietary patterns and obesity phenotypes. Int $\mathrm{J}$ Obes 2009; 33(12): 1419-26.

19. Tsai YT, Liu JP, Tu YK, Lee MS, Chen PR, Hsu HC, et al. Relationship between dietary patterns and serum uric acid concentrations among ethnic Chinese adults in Taiwan. Asia Pac J Clin Nutr 2012; 21(2): 263-70.

20. Choi HK, Curhan G. Beer, liquor, and wine consumption and serum uric acid level: the Third National Health and Nutrition Examination Survey. Arthritis Rheum 2004; 51(6): 1023-9.

21. Gan Y, Cao LS, Tian HM, Ran XW, Cheng T, Gao Y. The relationship of metabolic syndrome and hyperuricemia of Yi-people in Xichang city. J Southwest Med University 2010; 33(6): 634-6. Chinese.

22. Zhou AJ, Pan Q, Li AL, Qie YL, Wang QX, Gong Y. Predictive value of obesity and metabolism indexes for hyperuricemia among rural adult Yi residents in Liangshan region. Chinese $\mathbf{J}$ Public Health 2015; 31(12): 1646-50. Chinese

23. Liu B, Wang T, Zhao HN, Yue WW, Yu HP, Liu CX, et al. The prevalence of hyperuricemia in China: a meta-analysis. BMC Public Health 2011; 11(1): 1-10. 
24. Huang S, Liu X, Li H, Xu W, Jia H. Sex difference in the association of serum uric acid with metabolic syndrome and its components: a cross-sectional study in a Chinese Yi population. Postgrad Med 2017; 129(8): 828-33.

25. Nagahama K, Iseki K, Inoue T, Touma T, Ikemiya Y, Takishita $\mathrm{S}$. Hyperuricemia and cardiovascular risk factor clustering in a screened cohort in Okinawa, Japan. Hypertens Res 2004; 27(4): 227-33.

26. Alberti KG, Eckel RH, Grundy SM, Zimmet PZ, Cleeman JI, Donato KA, et al. Harmonizing the metabolic syndrome: a joint interim statement of the international diabetes federation task force on epidemiology and prevention; National heart, lung, and blood institute; American Heart Association; World Heart Federation; International Atherosclerosis Society; and International Association for the Study of Obesity. Circulation 2009; 120: $1640-5$

27. Zhang M, Chang H, Gao Y, Wang X, Xu W, Liu D, et al. Major dietary patterns and risk of asymptomatic hyperuricemia in Chinese adults. J Nutr Sci Vitaminol 2012; 58(5): 339-45.

28. Choi HK, Liu S, Curhan G. Intake of purine-rich foods, protein, and dairy products and relationship to serum levels of uric acid: the Third National Health and Nutrition Examination Survey. Arthritis Rheumatol 2005; 52(1): 283-9.

29. Xiong Z, Zhu C, Qian X, Zhu J, Wu Z, Chen L. Serum uric acid is associated with dietary and lifestyle factors in elderly women in suburban Guangzhou in Guangdong province of south China. J Nutr Health Aging 2013; 17(1): 30-4.

30. Villegas R, Xiang YB, Elasy T, Xu WH, Cai H, Cai Q, et al. Purine-rich foods, protein intake, and the prevalence of hyperuricemia: the Shanghai Men's Health Study. Nutr Metab Cardiovasc Dis 2012; 22(5): 409-16.
31. Choi HK, Atkinson K, Karlson EW, Willett W, Curhan G. Purine-rich foods, dairy and protein intake, and the risk of gout in men. N Engl J Med 2004; 350(11): 1093-103.

32. Krízek V. Serum uric acid in relation to body weight. Ann Rheum Dis 1966; 25(25): 456-8.

33. Carey DGP. Abdominal obesity. Curr Opin Lipidol 1998; 9(1): 35-40.

34. Kontogianni MD, Chrysohoou C, Panagiotakos DB, Tsetsekou E, Zeimbekis A, Pitsavos C, et al. Adherence to the Mediterranean diet and serum uric acid: the ATTICA study. Scand J Rheumatol 2012; 41(6): 442-9.

35. Li X, Song P, Li J, Wang P, Li G. Relationship between hyperuricemia and dietary risk factors in Chinese adults: a cross-sectional study. Rheumatol Int 2015; 35(12): 2079-89.

36. Huang HY, Appel LJ, Choi MJ, Gelber AC, Charleston J, Norkus EP, et al. The effects of vitamin C supplementation on serum concentrations of uric acid: results of a randomized controlled trial. Arthritis Rheum 2005; 52(6): 1843-7.

37. Gao X, Curhan G, Forman JP, Ascherio A, Choi HK. Vitamin $\mathrm{C}$ intake and serum uric acid concentration in men. $\mathrm{J}$ Rheumatol 2008; 35(9): 1853-8.

38. Sorensen LF. Gout secondary to chronic renal disease: studies on urate metabolism. Ann Rheum Dis 1980; 39(5): 424-30.

\section{*Hong Jia}

School of Public Health

Southwest Medical University

No. I, Section I, Xianglin road, Longmatan District

Luzhou Sichuan 646000, China

Tel: + |3088316|33.

Email:jhong_|z@|63.com 